07

\title{
Радиационно-индуцированный гальванический эффект, наблюдаемый в интерфейсе металл-диэлектрик
}

\author{
() В.И. Зайцев, И.А. Барыков, А.В. Карташов,
}

О.В. Терентьев, Н.Б. Родионов

Государственный научный центр „Троицкий институт инновационных и термоядерных исследований“, Москва, Троицк

E-mail: vzaitsev@triniti.ru

Поступило в Редакцию 21 июня 2016 г.

Исследуется эффект, наблюдающийся при взаимодействии электромагнитного излучения (энергия квантов $25-1000 \mathrm{eV}$ ) с диэлектриком, имеющим металлическое покрытие. Источником излучения служил мегаамперный Z-пинч. Измерения, проведенные с образцами оптического стекла, показали, что под действием излучения (мощность $\sim 10^{6} \mathrm{~W} / \mathrm{cm}^{2}$ ) в электрической цепи, включающей металлизированный диэлектрик, возникает ток. Авторы считают, что причиной данного гальванического эффекта является генерация в диэлектрике „горячих“ электронов.

Взаимодействие электромагнитного излучения с веществами имеет длинную историю исследований и широкую область практических применений. В области больших энергий квантов излучение является инструментом в различных областях рентгенографии и рентгеноскопии. Взаимодействия в области малых энергий квантов являются основой таких направлений, как когерентные излучатели (лазерная техника), фотоэлектрические преобразователи и др. В последнее время развитие новых технологий, как, например, рентгеновская литография, управляемый термоядерный синтез, стимулировало интерес к процессам, возникающим под действием электромагнитного излучения промежуточной области (жесткого ультрафиолетового и мягкого рентгеновского излучений), в частности к динамике электрических свойств материалов. Необходимо заметить, что, несмотря на создание мощных импульсных источников электромагнитного излучения, динамика 
электрических свойств твердых материалов, сопутствующая процессу интенсивного облучения, мало изучена. В работах $[1,2]$ исследовалось влияние тормозного излучения (энергия электронного пучка $600 \mathrm{keV}$ ) на электрические свойства таких типичных диэлектриков, как сапфир и оптическое стекло, которые благодаря уникальным свойствам (высокие прозрачность, твердость, радиационная стойкость) часто используются в качестве изолирующих и оптических материалов. Показано, что в процессе воздействия рентгеновского излучения в данных материалах возникает электропроводность, связанная с возникновением носителей в зоне проводимости. Получены значения основных параметров, необходимых для описания процесса возникающих гальванических явлений. Спектральный состав и интенсивность излучения в данных работах не приводятся. Работы, где исследуются процессы взаимодействия „мягкого“ излучения на электрические свойства изоляторов, нам неизвестны. В настоящей работе исследуются гальванические эффекты, возникающие в образцах аморфного оптического стекла под действием электромагнитного излучения большой мощности $\left(\sim 10^{6} \mathrm{~W} / \mathrm{cm}^{2}\right)$ в области энергий квантов $25-1000 \mathrm{eV}$.

Исследования проводились на термоядерной установке Ангара-5-1, где под действием мегаамперных токов (3-4 МА) на конечном этапе сжатия цилиндрических лайнеров образуется плазменное образование (Z-пинч), являющееся интенсивным источником мягкого рентгеновского излучения с длительностью импульса излучения $\sim 10 \mathrm{~ns}$ и полным выходом излучения до $50 \mathrm{~kJ}$ [3]. Спектральный состав излучения определяется параметрами лайнера (геометрия, атомный состав материала). Основные измерения проведены с W-лайнерами, состоящими из 40 проволок (диаметр 6-7 $\mu \mathrm{m}$ ), начально расположенных по окружности $12 \mathrm{~mm}$. В этом случае температура плазмы сжатого пинча составляет 60-70 eV. Спектральный состав излучения иллюстрирует рис. 1,a [4], где измеренный спектр сравнивается с „чернотельным“ излучением температурой $60 \mathrm{eV}$. Основная доля излучения находится в области энергий квантов менее $1 \mathrm{keV}$, глубина проникновения которых в стекло весьма мала, что можно видеть на рис. $1, b$, где показано также пропускание излучения с реальным спектральным составом оптическим стеклом различной толщины.

Исследуемые образцы диэлектриков (диэлектрические датчики) располагались в вакууме на расстоянии $2.5-3 \mathrm{~m}$ от источника рентгеновского излучения. Все измерения были проведены с образцами

Письма в ЖТФ, 2016, том 42, вып. 22 

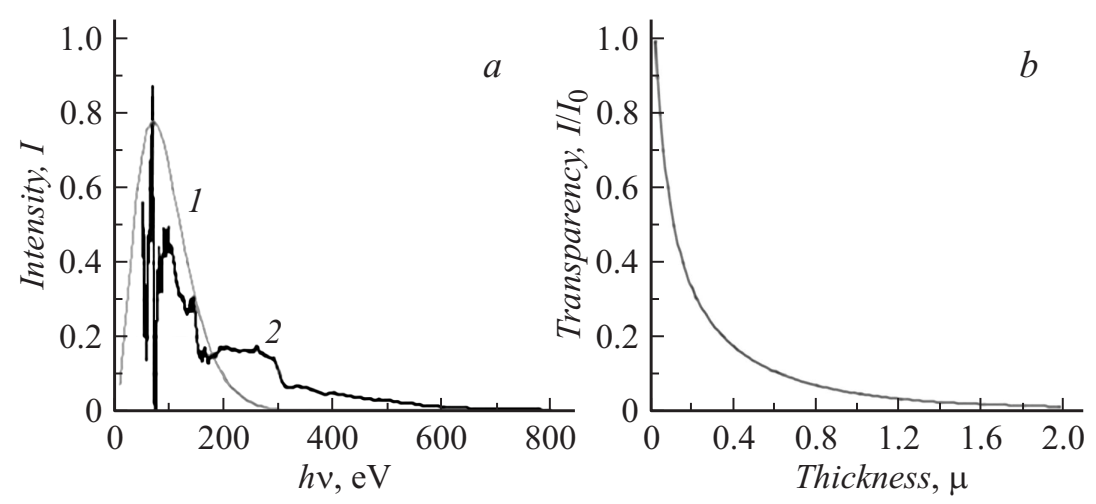

Рис. 1. $a-$ спектральный состав излучения Z-пинча: $1-$ измеренный спектр; 2 - спектр „чернотельного“ излучения с температурой $60 \mathrm{eV}$; материал лайнера $-\mathrm{W}$. Конструкция лайнера -40 проволок толщиной $7 \mu \mathrm{m}$ на диаметpe $12 \mathrm{~mm}$. Эксперимент № 4847. $b$ - зависимость прозрачности оптического стекла $\left(\mathrm{SiO}_{2}\right)$ от толщины образца для излучения со спектром W-пинча. В расчете использовались таблицы сайта http://henke.lbl.gov/optical_constants.

оптического стекла KU-1 площадью $S \approx 1 \mathrm{~cm}^{2}$, толщиной $L=500 \mu \mathrm{m}$ с двухсторонним Al-покрытием толщиной 300-500 А. Одновременно могли испытываться три образца с различными параметрами: тип материала, приложенное к кристаллам напряжение, входные фильтры. Конструкция датчиков предусматривала полную экранировку от влияния вторичной эмиссии с токоведущих элементов и продуктов ионизации остаточного газа на токовый сигнал детектора. Сигнал с детектора через коаксиальный кабель (длина $80 \mathrm{~m}$, волновое сопротивление $50 \Omega$ ) регистрировались осциллографом TDS 2024 (полоса частот регистрации $200 \mathrm{MHz}$ ). Схема измерений показана на рис. 2, $a$. Во всех измерениях одновременно с сигналами испытуемых образцов регистрировался сигнал вторично-эмиссионного детектора, который является штатным на установке. На рис. 2, $b$ приведены типичные сигналы отклика детекторов на падающее излучение с мощностью $\sim 2 \mathrm{MW} \cdot \mathrm{cm}^{-2}$. Можно видеть, что при данном уровне поглощаемой мощности величина отклика составляет $\sim 15 \mathrm{~V}$. Форма сигналов детекторов практически идентична, за исключением некоторых особенностей в начале и конце импульсов излучения, что мы связываем 
$a$

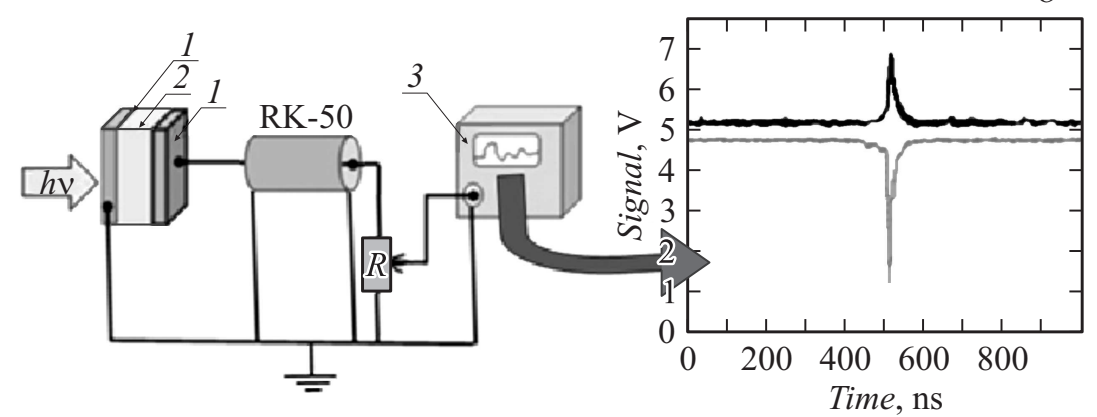

Pис. 2. $a-$ схема измерений: 1 - контактное Al-покрытие, толщина $300 \AA$; 2 - оптическое стекло, толщина $0.5 \mathrm{~mm} ; 3$ - осциллограф TDS 2024. $b$ - сигналы детекторов при облучении рентгеновским излучением Z-пинча установки Ангара-5-1 мощностью $2 \mathrm{MW} / \mathrm{cm}^{2}$ : нижний луч - диэлектрический детектор, верхний луч - вторично-эмиссионный детектор.

с различием спектральных характеристик вторично-эмиссионного и исследуемого диэлектрического датчиков. Отрицательная полярность сигнала диэлектрического датчика, возникающего на тыльной стороне образца, свидетельствует о возникновении положительного заряда на облучаемой стороне диэлектрика.

Необходимым условием возникновения в твердом теле гальванических эффектов является наличие свободных носителей заряда. Механизмом образования свободных носителей является перевод валентных электронов в зону проводимости, для чего требуется определенная энергия. Если в полупроводниках речь идет о затратах на образование пары носителей энергии около $1 \mathrm{eV}$, то в случае диэлектриков требуется энергия на порядки больше. Так, в [1,2] экспериментально показано, что в аморфном оптическом стекле на образование пары носителей необходимо потратить энергию $\Delta E \approx 150 \mathrm{eV}$. Одновременно в данных работах определены рекомбинационное время жизни образовавшихся электронов $\tau \approx 1.5 \cdot 10^{-8} \mathrm{~s}$.

В нашем случае образовавшиеся носители заряда локализуются в области $\Delta L \sim 1 \mu \mathrm{m}$ вблизи границы с контактным покрытием. Возникновение положительного заряда на облучаемой стороне диэлектрика

Письма в ЖТФ, 2016, том 42, вып. 22 
свидетельствует, что электронная компонента носителей достаточно быстро покидает зону поглощения излучения. Мы полагаем, что механизмом образования заряда являются процессы, обусловленные пространственными и температурными градиентами образовавшихся носителей [5], а именно диффузией электронов в результате разницы в значениях $n k T(n, T-$ плотность и температура электронов, $k$ - постоянная Больцмана) в металле контактного покрытия и образовавшихся в диэлектрике. Процесс весьма сходен с образованием контактной разницы потенциалов. Отличием является необходимость разницы температур электронов в двух средах и градиента плотности образовавшихся носителей заряда в диэлектрике („толстый“ образец). Сделаем некоторые оценки. Зная поглощенную в диэлектрике мощность излучения, полный заряд образовавшихся электронов можно записать в виде

$$
Q_{e}=\frac{W \tau}{\Delta E},
$$

где $W$ - поглощенная мощность; $\tau$-время жизни образовавшихся носителей, которое может определяться как рекомбинацией носителей, так и временем диффузионного дрейфа электронов к металлу покрытия.

Оценку $\tau$ можно сделать из следующих соображений. Диэлектрический датчик является конденсатором емкостью $C$, заряд которого $Q_{c}=U C$. Так как толщина области, занимаемая образовавшимися носителями $\Delta L \ll L$ ( $L-$ расстояние между обкладками), допустимо считать данную область одной из обкладок конденсатора. Сопоставляя $Q_{e}$ и $Q_{c}$ и используя значения экспериментально полученных напряжений, возникающих на образцах во время облучения $U=10-15 \mathrm{~V}$, получаем

$$
\tau=\frac{\varepsilon \varepsilon_{0} S U}{W L} \Delta E=\frac{(10-15) \cdot 8.85 \cdot 10^{-12} \cdot 150}{2 \cdot 10^{6} \cdot 500 \cdot 10^{-6}}=(0.12-0.14) \cdot 10^{-9} \mathrm{~s}
$$

где $c=\frac{\varepsilon \varepsilon_{0} S}{L}$.

Сравнение $\tau$ со значением, равным $(10-14) \cdot 10^{-9} \mathrm{~s}$, экспериментально полученным в [2], показывает, что в данном случае $\tau$ определяется градиентной диффузией электронов с последующей их потерей на металле покрытия. Таким образом, в результате быстрой диффузии образующихся электронов в сторону контактного покрытия на облучаемой стороне образца диэлектрика возникает положительный

Письма в ЖТФ, 2016, том 42, вып. 22 
заряд ионов, величина которого повторяет интенсивность падающего излучения. В том случае, если данная сторона образца находится под нулевым потенциалом, подключенная к нагрузке противоположная сторона принимает отрицательный потенциал, что можно видеть в приведенном сигнале на рис. $2, b$.

С целью оценки плотности возникающих в диэлектрике носителей $n_{d}$ примем, что область локализации возникающего заряда составляет примерно половину длины полного поглощения (рис. 2) $-0.5 \Delta L$. Тогда

$$
n_{d} \approx \frac{2 Q_{e}}{e S \Delta L}=1.8 \cdot 10^{18} \mathrm{~cm}^{-3}
$$

где $e-$ единичный заряд.

Так как плотность свободных электронов в $\mathrm{Al}$ при комнатных температурах $n_{\mathrm{Al}} \approx 10^{22} \mathrm{~cm}^{-3}$, то, следовательно, градиент электронной плотности не может быть причиной диффузии электронов из диэлектрика в металл покрытия.

Обратимся к известному выражению для контактной разницы потенциалов

$$
e \varphi=k T \ln \frac{n_{1}}{n_{2}} .
$$

Используя экспериментальные значения $\varphi=10-15 \mathrm{~V}$, получаем для температуры электронов в диэлектрике

$$
T[\mathrm{~K}] \approx \frac{e \varphi}{k \ln \frac{n_{\mathrm{Al}}}{n_{d}}} \approx \frac{1.6 \cdot 10^{-19}}{1.3 \cdot 10^{-23} \cdot 8.6} \varphi, \Rightarrow T=\frac{\varphi}{7.5},
$$

где $T-$ в еV, $\varphi-$ в V, т. е. наблюдаемый гальванический эффект может обеспечить температура образовавшегося в диэлектрике электронного „облака“ $T=1.3-2 \mathrm{eV}$. Необходимо иметь в виду, что данное выражение применяется для одинаковой температуры контактирующих материалов. В данном случае при оценке предполагается, что определяющую роль в процессе дрейфа носителей играют более „нагретые“ носители образовавшегося в диэлектрике электронного „облака“. Более строгий анализ наблюдаемого эффекта должен включать кинетику образования и гибели возникающих носителей заряда.

Экспериментальные исследования и анализ процессов взаимодействия электромагнитного излучения высокой мощности (область энергий квантов - ультрафиолет и мягкий рентген) показали, что в

Письма в ЖТФ, 2016, том 42, вып. 22 
электрических цепях и конструкциях, подвергающихся облучению, в интерфейсе металл-диэлектрик возникает разница потенциалов. Авторы считают, что причиной возникающего гальванического эффекта является сравнительно высокая температура образовавшихся в результате ионизации диэлектрика электронов. Необходимым условием возникновения эффекта, наряду с высокой температурой электронов, является наличие градиента плотности носителей заряда в диэлектрике („толстый“ поглотитель). Детальный анализ данного эффекта требует рассмотрения кинетики протекающих в интерфейсе процессов во время облучения. Одним из очевидных применений данного эффекта является разработка на его основе детекторов импульсных потоков рентгеновского излучения большой мощности, что является востребованным в исследованиях по управляемому термоядерному синтезу.

Авторы выражают благодарность своим коллегам: В.Е. Черковцу за постоянный интерес к данной работе и А.А. Самохину за ряд полезных советов в процессе исследований.

\section{Список литературы}

[1] Hugles R.C. // Phys. Rev. Lett. 1973. V. 30. N 26. P. 1333-1336.

[2] Hugles R.C. // Phys. Rev. Lett. 1979. V. 19. N 10. P. 5318-5328.

[3] Альбиков 3.А., Велихов Е.П., Веретенников А.И. и др. // Атомная энергия. 1990. T. 68. B. 1. C. 26-35.

[4] Болдарев А.С., Болховитинов Е.А., Вичев И.Ю. и др. // Физика плазмы. 2015. T. 41. № 2. C. 195-199.

[5] Белиндер В.И, Струман Б.И. // УФН. 1980. Т. 130. В. 3. С. 415-430. 